\title{
Recombinant human pentraxin-2 therapy in patients with idiopathic pulmonary fibrosis: safety, pharmacokinetics and exploratory efficacy
}

\author{
Bernt van den Blink ${ }^{1}$, Marlous R. Dillingh ${ }^{2}$, Leo C. Ginns ${ }^{3}$, Lake D. Morrison ${ }^{4}$, \\ Matthijs Moerland ${ }^{2}$, Marlies Wijsenbeek ${ }^{1}$, Elizabeth G. Trehu ${ }^{5}$, \\ Brian J. Bartholmai ${ }^{6}$ and Jacobus Burggraaf ${ }^{2}$
}

Affiliations: ${ }^{1}$ Dept of Pulmonary Medicine, Erasmus MC, Rotterdam, The Netherlands. ${ }^{2}$ Centre for Human Drug Research, Leiden, The Netherlands. ${ }^{3}$ Massachusetts General Hospital, Boston, MA, USA. ${ }^{4}$ Dept of Medicine, Duke University, Durham, NC, USA. ${ }^{5}$ Promedior, Inc., Lexington, MA, USA. ${ }^{6}$ Division of Thoracic Radiology, Mayo Clinic, Rochester, MI, USA.

Correspondence: Bernt van den Blink, Dept of Pulmonary Medicine Erasmus MC, 's-Gravendijkwal 230, Rotterdam, 3015 CE, The Netherlands. E-mail: B.vandenBlinkđerasmusmc.nl

ABSTRACT Abnormal fibrogenic repair response upon alveolar injury is believed to play an important role in the pathogenesis of idiopathic pulmonary fibrosis (IPF). PRM-151 (recombinant human pentraxin2, also known as serum amyloid P), has been shown to reduce fibrosis in preclinical lung fibrosis models, and was well tolerated with a favourable pharmacokinetic profile in an earlier single-dose phase I study.

A randomised, double-blind, placebo-controlled, multiple ascending dose trial was performed to assess the tolerability and pharmacokinetic and pharmacodynamic characteristics of multiple doses of PRM-151 in IPF patients. Subjects in three successive cohorts $\left(1,5\right.$, or $10 \mathrm{mg} \cdot \mathrm{kg}^{-1}$ versus placebo) received intravenous study drug on days $1,3,5,8$ and 15, and were followed-up to day 57 .

PRM-151 was well tolerated at all dose levels, with no serious adverse reactions. Administration of PRM151 resulted in two- to eight-fold dose-dependent increases in circulating pentraxin-2 levels. Forced vital capacity and 6-min walk test showed trends towards improvement in the combined PRM-151 dose groups. On high-resolution computed tomography scans, stable or improved lung volume unoccupied by interstitial lung abnormality was noted in some PRM-151 subjects compared to placebo subjects on day 57.

The efficacy of PRM-151 in IPF remains to be investigated in dedicated future trials.

@ERSpublications

Recombinant human pentraxin-2 increased serum levels safely in IPF patients and may improve lung function http://ow.ly/WaqDQ

This article has supplementary material available from erj.ersjournals.com

Received: May 292015 | Accepted after revision: Dec 072015 | First published online: Feb 122016

This study is registered at ClinicalTrials.gov with identifier number NCT01254409.

Support statement: This study was funded by Promedior, Inc. All authors received clinical trial support from Promedior, Inc. E.G. Trehu is an employee of Promedior, Inc. Funding information for this article has been deposited with FundRef.

Conflict of interest: Disclosures can be found alongside the online version of this article at erj.ersjournals.com

Copyright OERS 2016 


\section{Introduction}

Idiopathic pulmonary fibrosis (IPF) is a debilitating and fatal disease with a median survival time of 3 years upon diagnosis. Patients with IPF suffer from cough, progressive dyspnoea on exertion and lung function decline. Recent positive results in investigational drug trials have shown that IPF is amenable to therapy and reduction in lung function decline is attainable [1, 2]. However, patients using these new drugs still suffer from progressive disease and only lung transplantation offers a curative option to a limited number of patients, indicating the necessity for additional therapies $[3,4]$.

Although the pathogenesis of IPF has not been fully elucidated, an aberrant fibrogenic repair response upon alveolar injury seems to plays a central role [5]. Improving normal wound healing and repair is therefore of great interest in IPF. The endogenous blood plasma protein pentraxin (PTX)-2, also known as serum amyloid P, is believed to play a crucial role in regulating wound healing and fibrosis [6]. PTX-2 is closely related to PTX-1 (C-reactive protein); both are short pentraxins synthesised by the liver. PTX-2 is constitutively synthesised at steady-state levels, while PTX-1 is an acute-phase protein that can respond to inflammatory signalling by rapid increase in synthesis and circulating levels. A third member of the pentraxin family is PTX-3, which is also an acute-phase protein with a significant role in the orchestration of tissue repair and remodelling [7].

PTX-2 has been shown to accumulate at sites of fibrosis in animal models [8]. PTX-2 inhibits differentiation of monocytes into pro-inflammatory and pro-fibrotic macrophages and fibrocytes, while promoting their differentiation into regulatory macrophages [9]. This in turn promotes epithelial healing and resolution of inflammation and scarring. The constitutive level of production and consumption of PTX-2 at fibrotic sites is thought to result in the lower circulating levels of PTX-2 in fibrotic disease, as seen in IPF [8], end-stage renal disease [8] and nonalcoholic steatohepatitis [10].

PRM-151 is recombinant human PTX-2, produced in a Chinese hamster ovary cell expression system, which has been shown to reduce fibrosis in preclinical models of transforming growth factor- $\beta 1$ - and bleomycin-induced lung fibrosis, with durable effects up to 30 days after dosing $[9,11,12]$. The first clinical study with PRM-151, in which single intravenous doses varying from 0.1 to $20 \mathrm{mg} \cdot \mathrm{kg}^{-1}$ were administered to healthy volunteers and a small cohort of pulmonary fibrosis patients, demonstrated that PRM-151 was well tolerated and that the compound displayed a favourable pharmacokinetic profile [13].

The current study was designed to assess the safety and tolerability of multiple escalating intravenous doses of PRM-151, to determine its pharmacokinetic behaviour, and to explore its pharmacodynamic effects, including functional measures such as pulmonary function tests, 6-min walking distance, patientreported outcomes and plasma biomarkers relevant for the pathophysiology of pulmonary fibrosis. After study completion, a retrospective analysis of data from high-resolution computed tomography (HRCT) scans was performed using a quantitative imaging modality. Some of the results of these studies have been previously reported in the form of an abstract [14].

\section{Methods}

\section{Study design}

The study was a randomised, placebo-controlled, double-blind, sequential-group study of ascending multiple doses of PRM-151. The study was conducted in accordance with the Declaration of Helsinki and good clinical practice guidelines, and was approved by local ethical review boards. Three study centres (two in the USA and one in the Netherlands) enrolled a total of 21 patients. All subjects provided written informed consent prior to enrolment.

Subjects were enrolled in three successive cohorts, each to include seven subjects, randomly assigned to receive either placebo $(\mathrm{n}=2)$ or PRM-151 $\left(1,5\right.$ or $10 \mathrm{mg} \cdot \mathrm{kg}^{-1} ; \mathrm{n}=5$ per successive dose level). Each subject participated in the study for $\sim 13$ weeks, which included a screening evaluation within 35 days before the first test article administration and a study period of 8 weeks entailing two brief inpatient periods and 11 outpatient visits that could be conducted at home at the subject's request. The test article was administered on days $1,3,5,8$ and 15 as an i.v. infusion over $30 \mathrm{~min}$, under fasting conditions. The decision to progress to the next dose level was made following review of the blinded safety data up to day 6 for at least four subjects.

\section{Main subject inclusion and exclusion criteria}

Participants were to be males or females of non-childbearing potential, aged 40-80 years, with a body mass index of $18-33 \mathrm{~kg} \cdot \mathrm{m}^{-2}$ and a body weight $\geqslant 45 \mathrm{~kg}$. All subjects had a diagnosis of IPF as determined by a definite or possible usual interstitial pneumonia (UIP) pattern on HRCT as described in the current American Thoracic Society (ATS)/European Respiratory Society/Japanese Respiratory Society/Latin American Thoracic Association statement [4], or by a surgical lung biopsy consistent with IPF. Other idiopathic interstitial pneumonias or interstitial lung diseases associated with environmental exposure, 
medication or systemic disease were excluded. Other inclusion criteria included forced vital capacity (FVC) $\geqslant 45 \%$ predicted; diffusing capacity of the lung for carbon monoxide (DLCO) 35-80\%; and a resting oxygen saturation on oxygen $\geqslant 88 \%$. The use of cyclophosphamide, systemic steroids, immunosuppressives or any investigational drug within 35 days before test article administration was prohibited.

\section{Safety analysis}

Safety was evaluated from reported clinical adverse events, scheduled physical examinations, vital signs, 12-lead ECGs, HRCT findings and clinical laboratory test results. Serum levels of antibodies to PTX-2 were measured to evaluate for immune response on day 1 (pre-dose) and on days 29 and 57. All subjects were carefully observed for symptoms of infusion reaction, including allergic reactions or hypersensitivity (e.g. urticaria and pruritus).

\section{Pharmacokinetic analysis}

Blood samples were obtained pre-dose and at multiple time points on days 1 through 57 for pharmacokinetic analysis. Plasma PRM-151 concentrations were determined using a validated ELISA method (Intertek Pharmaceutical Services, San Diego, CA, USA). Noncompartmental pharmacokinetic analysis was performed for PRM-151 using Phoenix WinNonlin (version 6.3; Certara, Mountain View, CA, USA) and SigmaPlot (version 11; Systat Software, San Jose, CA, USA). Because the bioanalytical method did not differentiate between PRM-151 and endogenous human PTX-2, baseline PTX-2 concentration was subtracted from all post-dose concentrations to generate baseline-corrected PRM-151 plasma concentrations. Baseline-corrected PRM-151 values that were negative were assumed to be zero.

\section{Efficacy end-points}

The pharmacodynamic effects of PRM-151 were assessed using functional tests and measurement of relevant plasma biomarkers. Pulmonary function was assessed using pulmonary function tests (FVC, FVC $\%$ pred, forced expiratory volume in $1 \mathrm{~s}\left(\mathrm{FEV}_{1}\right)$ and $\left.\mathrm{DLCO}\right)$ at screening and on days 1,15 and 57 . A 6-min walk test (6MWT) was performed at screening and on days 16 and 57 according to the 2002 ATS standards. Subjects were also asked to complete the St George's Respiratory Questionnaire (SGRQ) on days 1, 16, 22 and 57. EDTA blood samples were collected over the course of the study period for assessment of a variety of blood biomarkers (including multiple matrix metalloproteinases, cytokines and chemoattractants) using either a custom glass slide array (53 biomarkers analysed by RayBiotech, Norcross, GA, USA) or an ELISA (four biomarkers analysed by Promedior, Lexington, MA, USA). Because of the limited sample size, only exploratory analyses were planned, allowing the identification of gross trends across treatment groups.

Noncontrast HRCT scans with contiguous reconstruction, performed according to local institutional protocols, were obtained at screening (4-5 weeks prior to day 1 ) and at day 57 . For each subject, the HRCT scan data with the highest contiguous reconstruction technique that were most similar at both time points were utilised for analysis. A retrospective exploratory quantitative analysis was performed centrally using CALIPER (computer-aided lung informatics for pathology evaluation and rating) software (developed by Mayo Clinic, Rochester, MN, USA and licensed to Imbio, Minneapolis, MN, USA). Lung texture analysis using CALIPER classifies each voxel of lung parenchyma based on morphology and density characteristics into one of five subtypes: normal; ground glass; reticular; honeycombing; and mild, moderate or severe low attenuation areas. In analysis of the extent of disease in this study, areas of ground glass, reticular and honeycombing subtypes were combined as interstitial lung abnormalities (ILA) and normal or mild low attenuation areas were considered non-ILA parenchyma (normal or mild lowattenuation areas representing hyperinflated tissue not involved by interstitial lung disease features). Previous studies have shown that CALIPER results are comparable to but more reproducible than radiologist assessment [15]; they correlate with FVC \% pred, DLCO and 6MWT [16]; and changes in ILA features over time are predictive of mortality in IPF [17]. CALIPER results were reported as change from screening to day 57 in percentage of total lung volume occupied by ILA and non-ILA features.

\section{Results}

Demographics

In total, 21 subjects were enrolled: eight subjects in cohort $1\left(1 \mathrm{mg} \cdot \mathrm{kg}^{-1}\right)$, seven subjects in cohort 2 $\left(5 \mathrm{mg} \cdot \mathrm{kg}^{-1}\right)$ and six subjects in cohort $3\left(10 \mathrm{mg} \cdot \mathrm{kg}^{-1}\right)$. All cohorts included two subjects treated with a placebo solution. In the first cohort, treatment was terminated for one subject due to observed adverse events (see later). This subject was replaced by another subject treated at the same dose level. In the last cohort, only six subjects were enrolled (PRM-151 $n=4$ and placebo $n=2$ ) due to limited supply of study medication. Demographics are presented in table 1 . In four subjects, all aged $>65$ years, a diagnosis of IPF was made based on possible UIP on HRCT without histology, in an appropriate clinical setting. Although according to 


\section{TABLE 1 Patient characteristics}

\begin{tabular}{|c|c|c|c|c|c|}
\hline & \multirow[t]{2}{*}{ Placebo } & \multicolumn{4}{|c|}{ PRM-151 } \\
\hline & & $1 \mathrm{mg} \cdot \mathrm{kg}^{-1}$ & $5 \mathrm{mg} \cdot \mathrm{kg}^{-1}$ & $10 \mathrm{mg} \cdot \mathrm{kg}^{-1}$ & All doses \\
\hline Subjects & 6 & 6 & 5 & 4 & 14 \\
\hline Age years & $65.5 \pm 12.9$ & $63.7 \pm 8.5$ & $70.6 \pm 8.3$ & $66.5 \pm 5.7$ & $66.7 \pm 7.8$ \\
\hline Male & $4(67)$ & $5(83)$ & $4(80)$ & $4(100)$ & 13 (87) \\
\hline \multicolumn{6}{|l|}{ Race } \\
\hline White & $5(83)$ & $6(100)$ & $5(100)$ & $4(100)$ & $15(100)$ \\
\hline African American & $1(17)$ & $0(0)$ & $0(0)$ & $0(0)$ & $0(0)$ \\
\hline BMI $\mathrm{kg} \cdot \mathrm{m}^{-2}$ & $28.7 \pm 3.1$ & $28.6 \pm 2.3$ & $28.6 \pm 4.6$ & $28.9 \pm 2.7$ & $28.7 \pm 3.1$ \\
\hline Oxygen & $4(67)$ & $0(0)$ & $1(20)$ & $0(0)$ & $1(7)$ \\
\hline \multicolumn{6}{|l|}{ UIP on HRCT } \\
\hline No & 2 (33) & $3(50)$ & $0(0)$ & $0(0)$ & $3(20)$ \\
\hline Possible & $1(17)$ & $1(17)$ & $2(40)$ & $2(50)$ & $5(33)$ \\
\hline Definite & $3(50)$ & 2 (33) & $3(60)$ & $2(50)$ & $7(47)$ \\
\hline UIP upon lung biopsy & $2(33)$ & $3(50)$ & $1(20)$ & $0(0)$ & $4(27)$ \\
\hline
\end{tabular}

Data are presented as $\mathrm{n}$, mean \pm SD or $\mathrm{n}(\%)$. BMI: body mass index; UIP: usual interstitial pneumonia; HRCT: high-resolution computed tomography.

current consensus a diagnosis of "unclassifiable idiopathic interstitial pneumonia" may be considered in these patients [18], several studies indicate that the diagnostic likelihood of IPF in such a setting is very high ( 95\%) $[19,20]$. Another subject did not have definite UIP on HRCT but was diagnosed with familial IPF; histology following subsequent lung transplantation showed a definite UIP pattern.

\section{Safety}

No infusion reactions, dose-limiting toxicity or serious adverse events were observed. In addition, no antibodies to PRM-151 were detected. In the PRM-151-treated subjects, the most common treatment-emergent adverse events recorded during the study were cough $(n=7,47 \%)$; productive cough $(n=4,27 \%)$; fatigue $(n=3,20 \%)$; and headache $(n=3,20 \%)$. The incidence of these events was comparable in the placebo group (cough 33\% $(n=2)$; productive cough $33 \%(n=2)$; fatigue $17 \%(n=1)$; and headache $17 \%(n=1)$ ) (table 2). Neither the nature nor the frequency of these reported adverse events increased with ascending PRM-151 dose levels. One subject in the $1 \mathrm{mg} \cdot \mathrm{kg}^{-1}$ dose group experienced an episode of moderate hypotension and dizziness just before administration of the third dose of PRM-151. These symptoms were considered possibly related to PRM-151 administration, and resulted in the discontinuation of PRM-151 treatment for that subject.

\section{Pharmacokinetics}

The mean \pm SEM baseline plasma concentration of PTX-2 was $41.2 \pm 3.4 \mu \mathrm{g} \cdot \mathrm{mL}^{-1}$ in the six placebo-treated subjects and $32.6 \pm 1.2 \mu \mathrm{g} \cdot \mathrm{mL}^{-1}$ in the 15 PRM-151-treated subjects. Administration of 1,5 and $10 \mathrm{mg} \cdot \mathrm{kg}^{-1}$ PRM-151 to IPF patients resulted in peak plasma concentrations that were two-, five- and eight-fold elevated compared to the mean baseline plasma PTX-2 levels (fig. 1a and b). Because of minimal circadian variability in endogenous PTX-2 (as evidenced by the values measured over time in the placebo-treated subjects (not shown)), noncompartmental analysis of PRM-151 pharmacokinetics was performed after subtraction of each individual subject's endogenous baseline PTX-2 level from levels at later time points. PRM-151 exposure (maximum concentration (Cmax) and area under the curve (AUC) over $48 \mathrm{~h}$ ) increased linearly with increasing doses (fig. $1 \mathrm{c}$ and d). Cmax was observed after 1-2 h. The median observed half-life on day 15 was $32.8 \mathrm{~h}$, but was highly variable between individual subjects, ranging from 11 to 110 h. PRM-151 did not accumulate in plasma when administered on a weekly basis (fig. 1a and b).

\section{Efficacy end-points}

Mean baseline pulmonary function measures (FVC, FVC \% pred, DLCO and FEV1) were lower in the placebo group compared to the PRM-151 groups (table 3). In the placebo group, FVC, FVC \% pred and FEV1 were all decreased on day 57 compared to baseline, indicative of a worsening of pulmonary function. In all PRM-151-treated groups, increased pulmonary function measures were observed compared to baseline levels, although the magnitude of increase in FVC, FVC \% pred and FEV1 was modest, and the differences between groups were not statistically significant. Relative increases of $\geqslant 5 \%$ in FVC $\%$ pred between baseline and day 57 occurred in six PRM-151-treated subjects (5\% increase in three subjects and 
TABLE 2 Adverse events

\begin{tabular}{|c|c|c|c|c|c|c|}
\hline & \multirow[t]{2}{*}{ Placebo } & \multicolumn{4}{|c|}{ PRM-151 } & \multirow[t]{2}{*}{ All } \\
\hline & & $1 \mathrm{mg} \cdot \mathrm{kg}^{-1}$ & $5 \mathrm{mg} \cdot \mathrm{kg}^{-1}$ & $10 \mathrm{mg} \cdot \mathrm{kg}^{-1}$ & All doses & \\
\hline Subjects & 6 & 6 & 5 & 4 & 15 & 21 \\
\hline Cough & 2 (33) & $4(67)$ & $3(60)$ & $0(0)$ & $7(47)$ & $9(43)$ \\
\hline Productive cough & $2(33)$ & $1(17)$ & $2(40)$ & $1(25)$ & $4(27)$ & 6 (29) \\
\hline Fatigue & $1(17)$ & $0(0)$ & $2(40)$ & $1(25)$ & $3(20)$ & $4(19)$ \\
\hline Headache & $1(17)$ & $2(33)$ & $0(0)$ & $1(25)$ & $3(20)$ & 4 (19) \\
\hline Dyspnoea & 2 (33) & 2 (33) & $0(0)$ & $0(0)$ & $2(13)$ & 4 (19) \\
\hline Dizziness & $1(17)$ & $1(17)$ & $1(20)$ & $0(0)$ & $2(13)$ & $3(14)$ \\
\hline Pruritus & $1(17)$ & $1(17)$ & $1(20)$ & $0(0)$ & $2(13)$ & $3(14)$ \\
\hline Haematoma & $1(17)$ & $2(33)$ & $0(0)$ & $0(0)$ & $2(13)$ & $3(14)$ \\
\hline Dyspnoea upon exertion & 2 (33) & $1(17)$ & $0(0)$ & $0(0)$ & $1(7)$ & $3(14)$ \\
\hline Nasopharyngitis & $2(33)$ & $1(17)$ & $0(0)$ & $0(0)$ & $1(7)$ & $3(14)$ \\
\hline Back pain & $2(33)$ & $0(0)$ & $1(20)$ & $0(0)$ & $1(7)$ & $3(14)$ \\
\hline Dysphonia & $0(0)$ & $2(33)$ & $0(0)$ & $0(0)$ & $2(13)$ & $2(10)$ \\
\hline Hypotension & $0(0)$ & $1(17)$ & $0(0)$ & $1(25)$ & $2(13)$ & $2(10)$ \\
\hline Lymphadenopathy & $1(17)$ & $1(17)$ & $0(0)$ & $0(0)$ & $1(7)$ & $2(10)$ \\
\hline Catheter site haematoma & $1(17)$ & $1(17)$ & $0(0)$ & $0(0)$ & 1 (7) & $2(10)$ \\
\hline Malaise & $1(17)$ & $1(17)$ & $0(0)$ & $0(0)$ & $1(7)$ & $2(10)$ \\
\hline Hypertension & $1(17)$ & $1(17)$ & $0(0)$ & $0(0)$ & $1(7)$ & $2(10)$ \\
\hline Noncardiac chest pain & 2 (33) & $0(0)$ & $0(0)$ & $0(0)$ & $0(0)$ & $2(10)$ \\
\hline Vomiting & $2(33)$ & $0(0)$ & $0(0)$ & $0(0)$ & $0(0)$ & $2(10)$ \\
\hline
\end{tabular}

Data are presented as $\mathrm{n}$ or $\mathrm{n}(\%)$. Events reported by at least two (10\%) out of the 21 subjects are listed. Treatment-emergent adverse events were defined as events occurring from the first day of dosing to the end of the study (day 57 or early termination day).

$10 \%$ increase in three subjects) and in none of the placebo-treated subjects (fig. 2). A relative decline of $\geqslant 5 \%$ from baseline FVC \% pred was observed in two PRM-151-treated subjects and two placebo subjects (fig. 2). No indication of PRM-151 dose level dependency on lung function tests was observed, and there was no apparent correlation between change in FVC \% pred and Cmax or AUC of PRM-151. PRM-151 treatment did not have an effect on DLCO.

Mean distance walked in the 6MWT decreased slightly over time in the placebo group and the lowest PRM-151 dose group ( $\left.1 \mathrm{mg} \cdot \mathrm{kg}^{-1}\right)$, and the mean distance walked improved in the two higher PRM-151 dose groups (table 3). None of these differences were statistically significant. No PRM-151 effect was observed on SGRQ outcome (not shown).

Plasma vascular endothelial growth factor levels progressively increased over time in the placebo-treated subjects; the levels remained stable in the PRM-151-treated subjects. Other plasma biomarkers potentially related to fibrosis or the biology of PTX-2, including matrix metalloproteinase (MMP)-7, intercellular adhesion molecule (ICAM)-1, interleukin (IL)-8, vascular cell adhesion molecule (VCAM)-1 and S100A12 did not show differences between PRM-151 and placebo groups (online supplementary table S1).

Baseline quantitative imaging data were analysed in 16 subjects who had $\leqslant 36$ days between screening HRCT and day 1 pulmonary function tests. There was a strong negative correlation between screening FVC $\%$ pred and percentage of lung volume identified as non-ILA using CALIPER ( $r=-0.7$; fig. 3 ), which demonstrates the potential value of quantitative imaging as biomarker in clinical trials in IPF. Non-ILA lung volume decreased in all placebo subjects and was stable or increased in five PRM-151-treated subjects, all of whom had stable or increased FVC \% pred (online supplementary table S2). There was no clear correlation between the magnitude of change in FVC \% pred and percentage of non-ILA lung volume (not shown). HRCT analysis was limited in four subjects by poor inspiratory effort on the day 57 scan. Non-ILA determination was possibly limited by the technical limitations of the retrospective analysis of the HRCT data, including differences in HRCT protocols used at the different study sites.

\section{Discussion}

This study demonstrated that PRM-151, administered as five i.v. infusions over 15 days at doses ranging from 1 to $10 \mathrm{mg} \cdot \mathrm{kg}^{-1}$, was well tolerated in patients with IPF, and did not raise any safety concerns. One subject receiving $1 \mathrm{mg} \cdot \mathrm{kg}^{-1}$ PRM-151 experienced an episode of moderate hypotension and dizziness, which resulted in treatment termination prior to the third dose. This event was considered possibly to be 

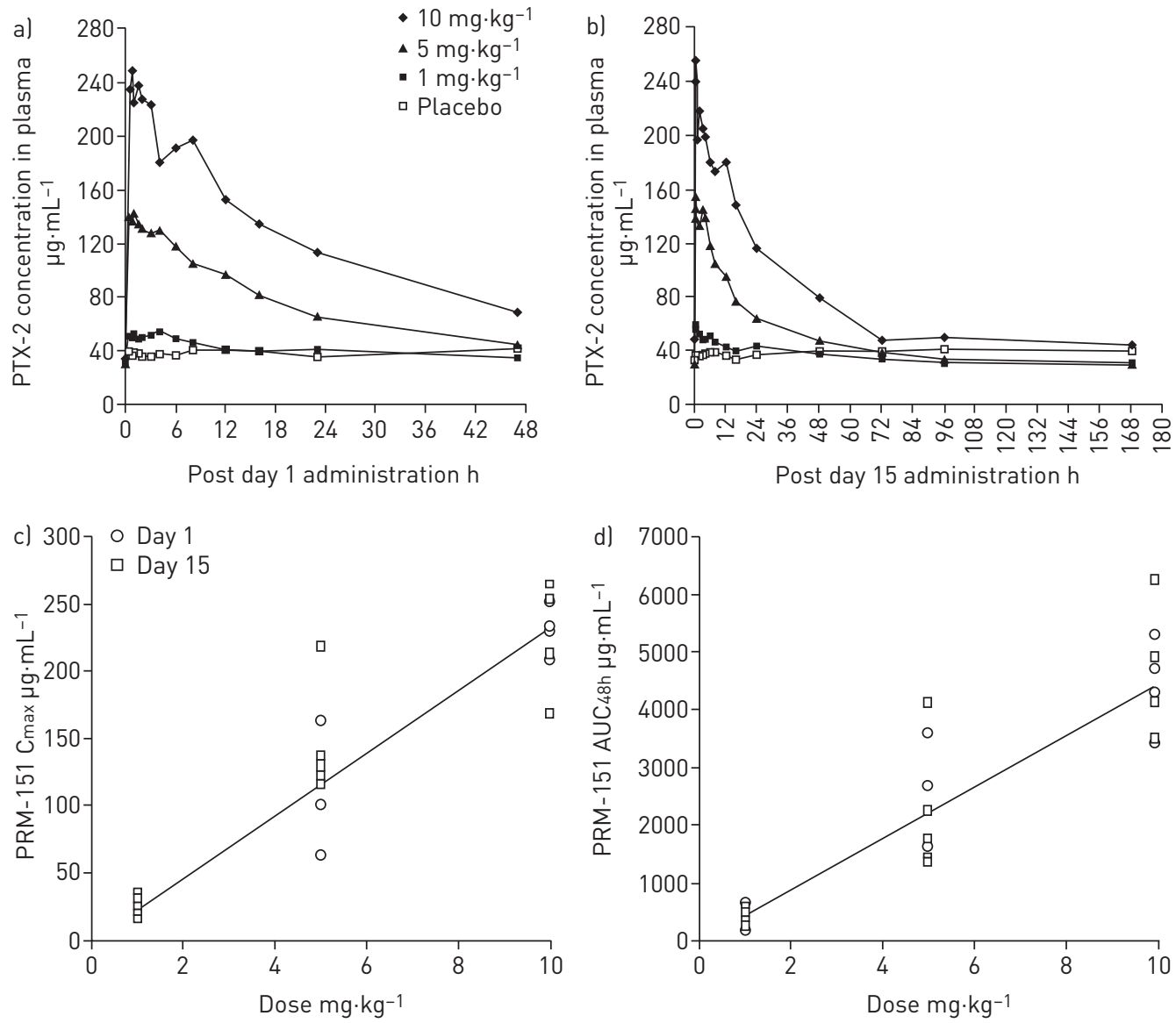

FIGURE 1 a) and b) Mean plasma concentration of pentraxin (PTX)-2 versus time. Time course over a) $48 \mathrm{~h}$ following the first dose of PRM-151 on day 1 ; b) $170 \mathrm{~h}$ following the last dose of PRM-151 on day 15. Lower limit of quantitation $4.0 \mathrm{ng} \cdot \mathrm{mL}^{-1}$. Individual subject values for $\mathrm{c}$ ) maximum concentration ( $C_{\text {max }}$ ) and d) area under the curve at $48 \mathrm{~h}$ (AUC48h) versus PRM-151 dose following day 1 and day 15 administrations. All values are based on baseline-corrected PTX-2 plasma concentrations.

TABLE 3 Pulmonary function tests and 6-min walking distance (6MWD): baseline values and change from baseline on day 57

Placebo

PRM-151

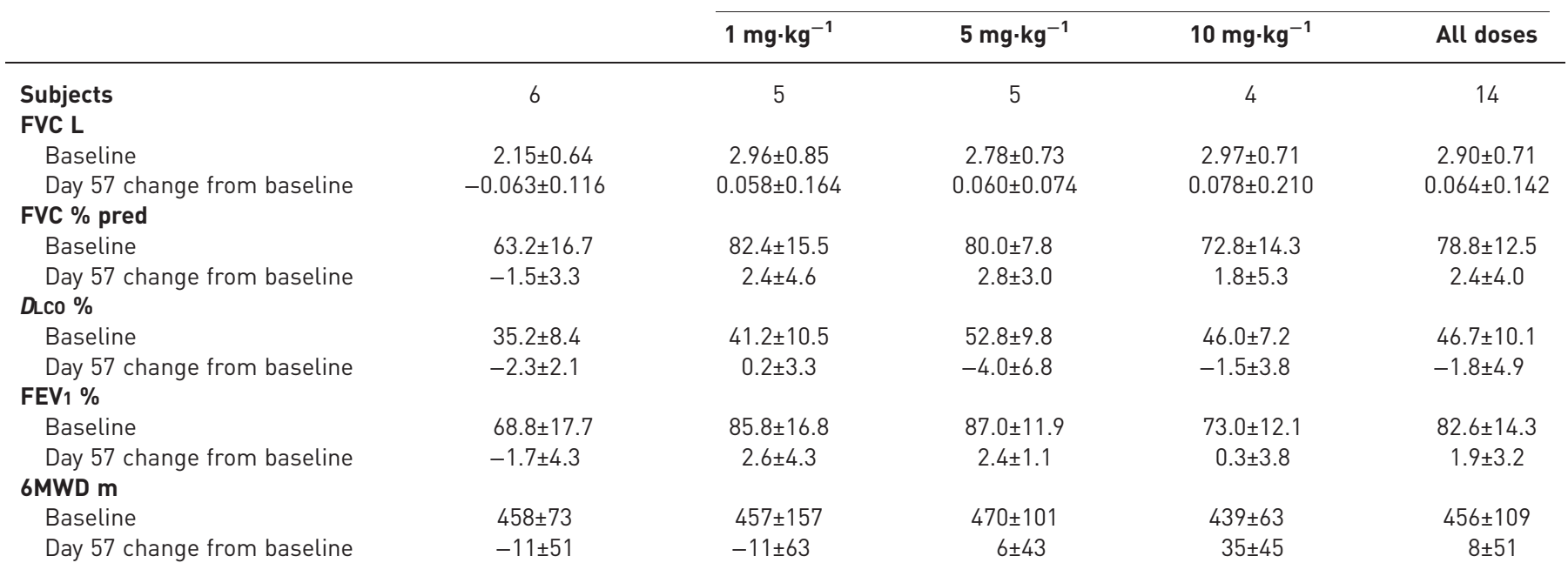

Data are presented as $\mathrm{n}$ or mean£SD. FVC: forced vital capacity; DLCO: diffusing capacity of the lung for carbon monoxide; FEV1: forced expiratory volume in $1 \mathrm{~s}$. 


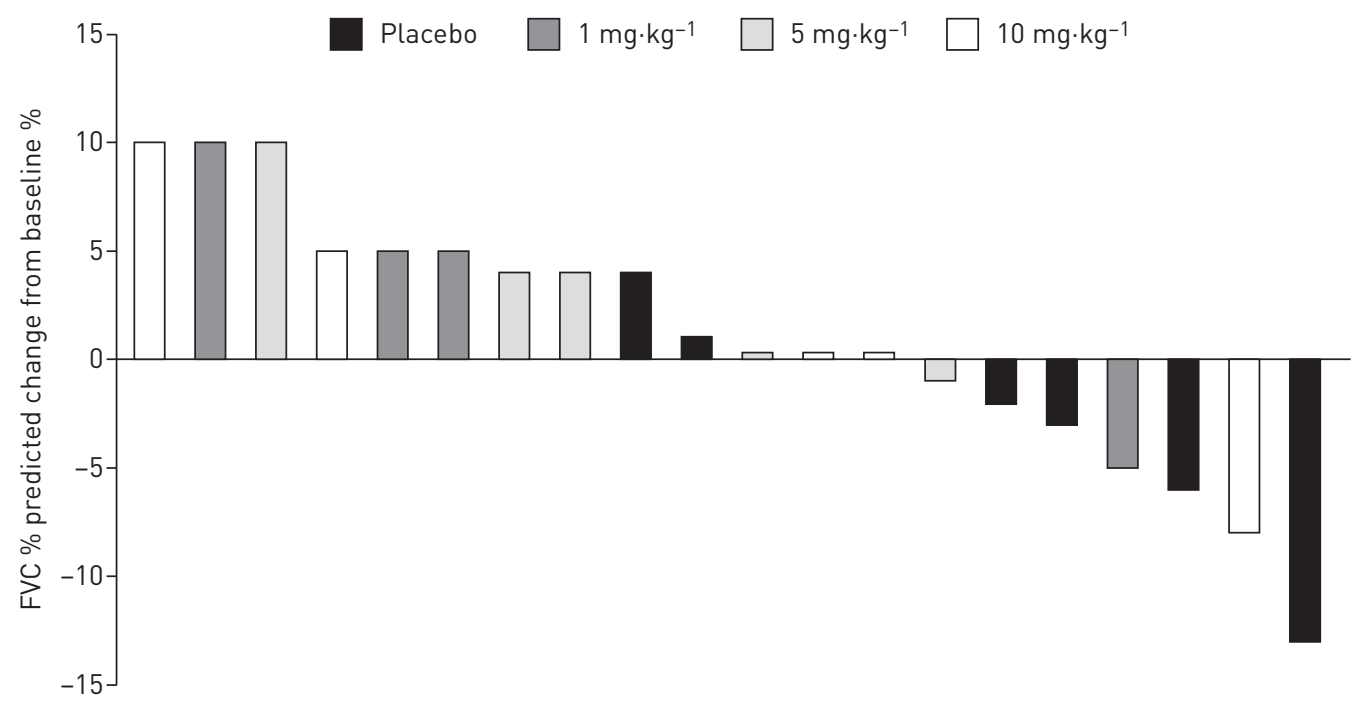

FIGURE 2 Individual subject values of relative change in forced vital capacity (FVC) \% predicted from baseline to day 57.

related to PRM-151 administration. The most frequently reported adverse events (cough, productive cough, fatigue and headache) were equally distributed between PRM-151- and placebo-treated subjects, suggesting that these events were not related to the test article and that some (such as cough) were a natural part of the disease. Neither the nature nor the frequency of these adverse events increased with ascending PRM-151 dose levels. Importantly, no allergic or anaphylactic signs were observed.

At $10 \mathrm{mg} \cdot \mathrm{kg}^{-1}$, the highest dose level tested, administration of PRM-151 resulted in an eight-fold increase in circulating PTX-2 levels, which remained elevated for $\geqslant 2$ days. These data confirm the earlier observations on PRM-151 kinetics in IPF patients [13]. There were no indications for accumulation of PRM-151 in plasma when administered at the selected dosing regimen. The interindividual variability observed in half-life was substantial, which may relate to the small and heterogeneous study population (e.g. sex and disease state). A population pharmacokinetic approach may be suitable for the identification of key covariates determining the pharmacokinetic behaviour of PRM-151, which may be supportive for further clinical development of the compound.

Based on preclinical experiments in animal models, PRM-151 was expected to exert pharmacological activity at the dose levels explored in this clinical study. In mouse and rat models of lung fibrosis, purified mouse and rat PTX-2 were effective at dose levels of $2.5 \mathrm{mg} \cdot \mathrm{kg}^{-1}$ and $1.6 \mathrm{mg} \cdot \mathrm{kg}^{-1}$, respectively, while human PTX-2 was routinely effective at $2-10 \mathrm{mg} \cdot \mathrm{kg}^{-1}$ in the mouse models of lung fibrosis (no lower doses were evaluated) $[9,11,12,21]$. Although the physiological validity and translatability of animal fibrosis models to human IPF can be argued [22], these animal study results indicate sufficient PRM-151 exposure for intended pharmacological effect at the dose levels of $1-10 \mathrm{mg} \cdot \mathrm{kg}^{-1}$ selected in our clinical study. PTX-2 acts as a ligand for Fc $\gamma$ receptors expressed on monocytes and macrophages, stimulating their differentiation into restorative regulatory macrophages with their local production of IL-10 [8]. Therefore, the optimal dose regimen of PRM-151 may require a threshold dosing interval to effectively

FIGURE 3 Correlation between screening values of percentage of lung volume identified as interstitial lung abnormality (ILA) and forced vital capacity (FVC) \% predicted.

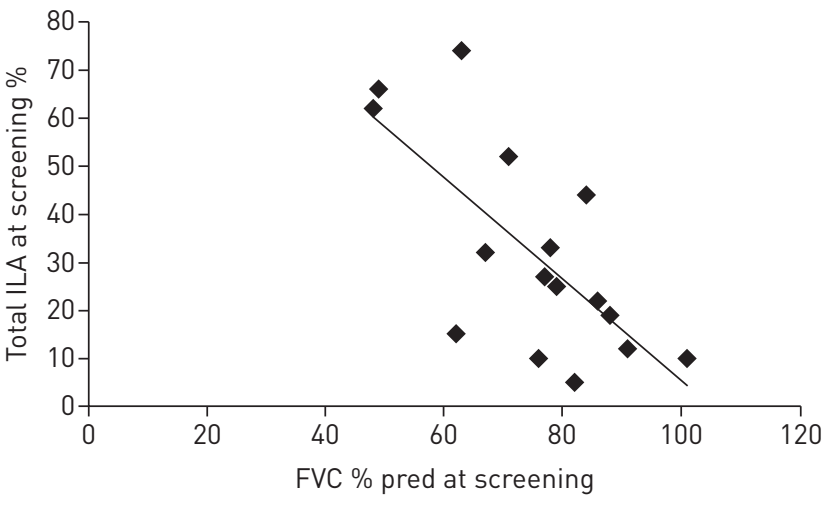


modulate tissue-resident monocyte/macrophage populations. Although our study was not designed to demonstrate clinical efficacy of PRM-151, potential trends in clinical effects of PRM-151 were explored that could guide further clinical development of the compound. Some trends towards improvement in multiple pulmonary function measures (FVC, FVC \% pred, FEV 1 and 6-min walking distance) and increases in normal lung volume by quantitative imaging on day 57 were observed. We cannot exclude the possibility that the observed trend for PRM-151 effects was related to differences in disease severity between placebo and PRM-151 treatment groups, despite randomisation and the double-blind nature of the study. Plasma biomarkers (MMP-7, IL-10, ICAM-1, IL-8, VCAM-1 and S100A12), validated to indicate disease prognosis, did not show differences between PRM-151 and placebo groups [23]. Since the sample size in the clinical study was small, and the treatment duration was relatively short, no firm conclusions can be drawn on clinical efficacy end-points. Evaluation of cellular biomarkers at the site of disease, such as alveolar macrophages, would have been valuable for assessment of the biological effect of PRM-151. The repeated investigations necessary to obtain such biomarkers in a study population burdened by considerable disease were deemed unfeasible for this study. However, it should be noted that such an approach is being employed in an IPF trial (clinicaltrials.gov NCT01371305).

In summary, five i.v. doses of PRM-151 over 2 weeks were generally well tolerated in IPF patients. Administration of PRM-151 resulted in a substantial increase in circulating PTX-2 levels. Some clinical efficacy markers showed trends towards improvement in the PRM-151 dose groups. However, efficacy of PRM-151 in IPF will be investigated in a dedicated future trial, which will prospectively evaluate both changes in lung function and tissue abnormalities by quantitative image analysis of HRCT.

\section{Acknowledgements}

The authors would like to thank Christiane Baud (independent medical writer, Dallas, TX, USA) for assistance in preparing the manuscript.

\section{References}

1 King TE Jr, Bradford WZ, Castro-Bernardini S, et al. A phase 3 trial of pirfenidone in patients with idiopathic pulmonary fibrosis. N Engl J Med 2014; 370: 2083-2092.

2 Richeldi L, du Bois RM, Raghu G, et al. Efficacy and safety of nintedanib in idiopathic pulmonary fibrosis. $N$ Engl J Med 2014; 370: 2071-2082.

3 King TE Jr, Brown KK, Raghu G, et al. BUILD-3: a randomized, controlled trial of bosentan in idiopathic pulmonary fibrosis. Am J Respir Crit Care Med 2011; 184: 92-99.

4 Raghu G, Collard HR, Egan JJ, et al. An official ATS/ERS/JRS/ALAT statement: idiopathic pulmonary fibrosis: evidence-based guidelines for diagnosis and management. Am J Respir Crit Care Med 2011; 183: 788-824.

5 Wolters PJ, Collard HR, Jones KD. Pathogenesis of idiopathic pulmonary fibrosis. Annu Rev Pathol 2014; 9: 157-179.

6 Cox N, Pilling D, Gomer RH. Serum amyloid P: a systemic regulator of the innate immune response. J Leukoc Biol 2014; 96: 739-743.

7 Doni A, Musso T, Morone D, et al. An acidic microenvironment sets the humoral pattern recognition molecule PTX3 in a tissue repair mode. J Exp Med 2015; 212: 905-925.

8 Castaño AP, Lin SL, Surowy $\mathrm{T}$, et al. Serum amyloid $\mathrm{P}$ inhibits fibrosis through FcyR-dependent monocyte-macrophage regulation in vivo. Sci Transl Med 2009; 1: 5 ra13.

9 Pilling D, Roife D, Wang M, et al. Reduction of bleomycin-induced pulmonary fibrosis by serum amyloid $\mathrm{P}$. J Immunol 2007; 179: 4035-4044.

10 Verna EC, Patel J, Bettencourt R, et al. Novel association between serum pentraxin-2 levels and advanced fibrosis in well-characterised patients with non-alcoholic fatty liver disease. Aliment Pharmacol Ther 2015; 42: 582-590.

11 Murray LA, Rosada R, Moreira AP, et al. Serum amyloid P therapeutically attenuates murine bleomycin-induced pulmonary fibrosis via its effects on macrophages. PLoS One 2010; 5: e9683.

12 Murray LA, Chen Q, Kramer MS, et al. TGF-beta driven lung fibrosis is macrophage dependent and blocked by serum amyloid P. Int J Biochem Cell Biol 2011; 43: 154-162.

13 Dillingh MR, van den Blink B, Moerland $\mathrm{M}$, et al. Recombinant human serum amyloid $\mathrm{P}$ in healthy volunteers and patients with pulmonary fibrosis. Pulm Pharmacol Ther 2013; 26: 672-676.

14 van den Blink B, Burggraaf J, Morrison LB, et al. A phase I study of PRM-151 in patients with Idiopathic Pulmonary Fibrosis. American Thoracic Society International Conference, May 17-22, 2013, Philadelphia, PA, USA. 2013: 5707.

15 Zavaletta VA, Bartholmai BJ, Robb RA. High resolution multidetector CT-aided tissue analysis and quantification of lung fibrosis. Acad Radiol 2007; 14: 772-787.

16 Raghunath S, Rajagopalan S, Karwoski RA, et al. Quantitative stratification of diffuse parenchymal lung diseases. PLoS One 2014; 9: e93229.

17 Maldonado F, Moua T, Rajagopalan S, et al. Automated quantification of radiological patterns predicts survival in idiopathic pulmonary fibrosis. Eur Respir J 2014; 43: 204-212.

18 Travis WD, Costabel U, Hansell DM, et al. An official American Thoracic Society/European Respiratory Society statement: update of the international multidisciplinary classification of the idiopathic interstitial pneumonias. Am J Respir Crit Care Med 2013; 188: 733-748.

19 Fell CD, Martinez FJ, Liu LX, et al. Clinical predictors of a diagnosis of idiopathic pulmonary fibrosis. Am J Respir Crit Care Med 2010; 181: 832-837.

20 Raghu G, Chen SY, Yeh WS, et al. Idiopathic pulmonary fibrosis in US Medicare beneficiaries aged 65 years and older: incidence, prevalence, and survival, 2001-11. Lancet Respir Med 2014; 2: 566-572. 
21 Pilling D, Buckley CD, Salmon M, et al. Inhibition of fibrocyte differentiation by serum amyloid P. J Immunol 2003; 171: 5537-5546.

22 Moore B, Lawson WE, Oury TD, et al. Animal models of fibrotic lung disease. Am J Respir Cell Mol Biol 2013; 49: 167-179.

23 Richards TJ, Kaminski N, Baribaud F, et al. Peripheral blood proteins predict mortality in idiopathic pulmonary fibrosis. Am J Respir Crit Care Med 2012; 185: 67-76. 